\title{
Fractional coloring of triangle-free planar graphs*
}

\author{
Zdeněk Dvořák ${ }^{\dagger}$ \\ Computer Science Institute of Charles University \\ Prague, Czech Republic \\ rakdver@iuuk.mff.cuni.cz \\ Jean-Sébastien Sereni ${ }^{\ddagger}$ \\ Centre National de la Recherche Scientifique (LORIA) \\ Nancy, France \\ sereni@kam.mff.cuni.cz \\ Jan Volec ${ }^{\S}$ \\ Mathematics Institute and DIMAP \\ University of Warwick \\ Coventry, UK \\ honza@ucw.cz
}

Submitted: Feb 22, 2014; Accepted: Oct 1, 2015; Published: Oct 16, 2015

Mathematics Subject Classifications: 05C15, 05C72

\begin{abstract}
We prove that every planar triangle-free graph on $n$ vertices has fractional chromatic number at most $3-\frac{3}{3 n+1}$.
\end{abstract}

\section{Introduction}

The interest in the chromatic properties of triangle-free planar graphs originated with Grötzsch's theorem [6], stating that such graphs are 3-colorable. Since then, several simpler proofs have been given, e.g., by Thomassen [13, 14]. Algorithmic questions have also been addressed: while most proofs readily yield quadratic algorithms to 3-color such graphs, it takes considerably more effort to obtain asymptotically faster algorithms. Kowalik [10] proposed an algorithm running in time $O(n \log n)$, which relies on the design of an

\footnotetext{
*This research was supported by the Czech-French Laboratory LEA STRUCO.

${ }^{\dagger}$ Supported by the Center of Excellence - Inst. for Theor. Comp. Sci., Prague, project P202/12/G061 of Czech Science Foundation.

¥This author’s work was partially supported by the French Agence Nationale de la Recherche under reference ANR 10 JCJC 020401.

$\S$ This author's work was supported by a grant of the French Government.
} 
advanced data structure. More recently, Dvořák, Kawarabayashi and Thomas [2] managed to obtain a linear-time algorithm, yielding at the same time a yet simpler proof of Grötzsch's theorem.

The fact that all triangle-free planar graphs admit a 3-coloring implies that all such graphs have an independent set containing at least one third of the vertices. Albertson, Bollobás and Tucker [1] had conjectured that there is always a larger independent set, which was confirmed by Steinberg and Tovey [12] even in a stronger sense: all triangle-free planar $n$-vertex graphs admit a 3-coloring where not all color classes have the same size, and thus at least one of them forms an independent set of size at least $\frac{n+1}{3}$. This bound turns out to be tight for infinitely many triangle-free graphs, as Jones [8] showed. As an aside, let us mention that the graphs built by Jones have maximum degree 4: this is no coincidence as Heckman and Thomas later established that all triangle-free planar $n$-vertex graphs with maximum degree at most 3 have an independent set of order at least $\frac{3 n}{8}$, which again is a tight bound - actually attained by planar graphs of girth 5 .

All these considerations naturally lead us to investigate the fractional chromatic number $\chi_{f}$ of triangle-free planar graphs. Indeed, this invariant is known to correspond to a weighted version of the independence ratio. In addition, since $\chi_{f}(G) \leqslant \chi(G)$ for every graph $G$, Grötzsch's theorem implies that $\chi_{f}(G) \leqslant 3$ whenever $G$ is triangle-free and planar. On the other hand, Jones's construction shows the existence of triangle-free planar graphs with fractional chromatic number arbitrarily close to 3 . Thus one wonders whether there exists a triangle-free planar graph with fractional chromatic number exactly 3 . Let us note that this happens for the circular chromatic number $\chi_{c}$, which is a different relaxation of the ordinary chromatic number such that $\chi_{f}(G) \leqslant \chi_{c}(G) \leqslant \chi(G)$ for every graph $G$.

The purpose of this work is to answer this question. We do so by establishing the following upper bound on the fractional chromatic number of triangle-free planar $n$-vertex graphs, which depends on $n$.

Theorem 1. Every planar triangle-free graph on $n$ vertices has fractional chromatic number at most $\frac{9 n}{3 n+1}=3-\frac{3}{3 n+1}$.

A consequence of Theorem 1 is that no (finite) triangle-free planar graph has fractional chromatic number equal to 3 . How much is it possible to improve the bound of Theorem 1 ? The aforementioned construction of Jones [8] yields, for each $n \geqslant 2$ such that $n \equiv 2$ $(\bmod 3)$, a triangle-free planar graph $G_{n}$ with $\alpha\left(G_{n}\right)=\frac{n+1}{3}$. Consequently, $\chi_{f}\left(G_{n}\right) \geqslant$ $\frac{3 n}{n+1}=3-\frac{3}{n+1}$. Therefore, the bound of form $3-\frac{c}{n}$ for some $c$ in Theorem 1 is qualitatively the best possible.

The bound can be improved for triangle-free planar graphs with maximum degree at most four, giving an exact result for such graphs.

Theorem 2. Every planar triangle-free n-vertex graph of maximum degree at most four has fractional chromatic number at most $\frac{3 n}{n+1}$.

Furthermore, the graphs of Jones's construction contain a large number of separating 4cycles (actually, all their faces have length five). We show that planar triangle-free graphs 
of maximum degree 4 and without separating 4-cycles cannot have fractional chromatic number arbitrarily close to 3 .

Theorem 3. There exists $\delta>0$ such that every planar triangle-free graph of maximum degree at most four and without separating 4-cycles has fractional chromatic number at most $3-\delta$.

Dvořák and Mnich [5] proved that there exists $\beta>0$ such that all planar triangle-free $n$-vertex graphs without separating 4-cycles contain an independent set of size at least $n /(3-\beta)$. This gives evidence that the restriction on the maximum degree in Theorem 3 might not be necessary.

Conjecture 4. There exists $\delta>0$ such that every planar triangle-free graph without separating 4-cycles has fractional chromatic number at most $3-\delta$.

Faces of length four are usually easy to deal with in the proofs by collapsing; thus the following seemingly simpler variant of Conjecture 4 is likely to be equivalent to it.

Conjecture 5 (Dvořák and Mnich [5]). There exists $\delta>0$ such that every planar graph of girth at least five has fractional chromatic number at most $3-\delta$.

\section{$2 \quad$ Notation and auxiliary results}

Consider a graph $G$. For an integer $a \geqslant 1$, let $[a]=\{1, \ldots, a\}$. An a-fractional coloring of $G$ is a function $\varphi$ assigning to each vertex of $G$ a subset of $[a]$, such that $\varphi(u) \cap \varphi(v)=\emptyset$ for all edges $u v$ of $G$. Let $f: V(G) \rightarrow[a]$ be any function. If the $a$-fractional coloring $\varphi$ satisfies $|\varphi(v)| \geqslant f(v)$ for every $v \in V(G)$, then $\varphi$ is an $(a, f)$-coloring of $G$. If $|\varphi(v)|=f(v)$ for every $v \in V(G)$, then the $(a, f)$-coloring $\varphi$ is tight. Note that if $G$ has an $(a, f)$-coloring, then it also has a tight one. If $f$ is the constant function assigning to each vertex of $G$ the value $b \in[a]$, then an $(a, f)$-coloring is said to be an $(a: b)$-coloring. An $a$-coloring is an $(a: 1)$-coloring.

Let $f_{1}: V(G) \rightarrow\left[a_{1}\right]$ and $f_{2}: V(G) \rightarrow\left[a_{2}\right]$ be arbitrary functions, and let $f: V(G) \rightarrow$ $\left[a_{1}+a_{2}\right]$ be defined by $f(v)=f_{1}(v)+f_{2}(v)$ for all $v \in V(G)$. Suppose that $\varphi_{i}$ is an $\left(a_{i}, f_{i}\right)$ coloring of $G$ for $i \in\{1,2\}$. Let $\varphi$ be defined by setting $\varphi(v)=\varphi_{1}(v) \cup\left\{a_{1}+c: c \in \varphi_{2}(v)\right\}$ for every $v \in V(G)$. Then $\varphi$ is an $\left(a_{1}+a_{2}, f\right)$-coloring of $G$, and we write $\varphi=\varphi_{1}+\varphi_{2}$. For an integer $k \geqslant 1$, we define $k \varphi$ to be $\underbrace{\varphi+\cdots+\varphi}_{k \text { times }}$.

The fractional chromatic number of a graph can be expressed in various equivalent ways, see [11] for details. In this paper, we use the following definition. The fractional chromatic number of $G$ is

$$
\chi_{f}(G)=\inf \left\{\frac{a}{b}: G \text { has an }(a: b) \text {-coloring }\right\} .
$$

We need several results related to Grötzsch's theorem. The following lemma was proved for vertices of degree at most three by Steinberg and Tovey [12]. The proof for vertices of degree four follows from the results of Dvořák and Lidický [4], as observed by Dvořák, Král' and Thomas [3]. 
Lemma 6. If $G$ is a triangle-free planar graph and $v$ is a vertex of $G$ of degree at most four, then there exists a 3-coloring of $G$ such that all neighbors of $v$ have the same color.

In fact, Dvořák, Král' and Thomas [3] proved the following stronger statement.

Lemma 7. There exists an integer $D \geqslant 4$ with the following property. Let $G$ be a trianglefree planar graph without separating 4-cycles and let $X$ be a set of vertices of $G$ of degree at most four. If the distance between every two vertices in $X$ is at least $D$, then there exists a 3-coloring of $G$ such that all neighbors of vertices of $X$ have the same color.

Let $G$ be a triangle-free plane graph. A 5 -face $f=v_{1} v_{2} v_{3} v_{4} v_{5}$ of $G$ is safe if $v_{1}, v_{2}$, $v_{3}$ and $v_{4}$ have degree exactly three, their neighbors $x_{1}, \ldots, x_{4}$ (respectively) not incident with $f$ are pairwise distinct and non-adjacent, and

- the distance between $x_{2}$ and $v_{5}$ in $G-\left\{v_{1}, v_{2}, v_{3}, v_{4}\right\}$ is at least four, and

- $G-\left\{v_{1}, v_{2}, v_{3}, v_{4}\right\}$ contains no path of length exactly three between $x_{3}$ and $x_{4}$.

Lemma 8 (Dvořák, Kawarabayashi and Thomas [2, Lemma 2.2]). If $G$ is a plane trianglefree graph of minimum degree at least three and all faces of $G$ have length five, then $G$ has a safe face.

Finally, let us recall the folding lemma, which is frequently used in the coloring theory of planar graphs.

Lemma 9 (Klostermeyer and Zhang [9]). Let $G$ be a planar graph with odd-girth at least $g>3$. If $C=v_{0} v_{1} \ldots v_{r-1}$ is a facial circuit of $G$ with $r \neq g$, then there is an integer $i \in\{0, \ldots, r-1\}$ such that the graph $G^{\prime}$ obtained from $G$ by identifying $v_{i-1}$ and $v_{i+1}$ (where indices are taken modulo $r$ ) is also of odd-girth at least $g$.

\section{Proofs}

First, let us show a lemma based on the idea of Hilton et al. [7].

Lemma 10. Let $G$ be a planar triangle-free graph. For a vertex $v \in V(G)$, let $f_{v}: V(G) \rightarrow$ [3] be defined by $f_{v}(v)=2$ and $f_{v}(w)=1$ for $w \in V(G) \backslash\{v\}$. If $v$ has degree at most 4 , then $G$ has a $\left(3, f_{v}\right)$-coloring.

Proof. Lemma 6 implies that there exists a 3-coloring of $G$ such that all neighbors of $v$ have the same color, without loss of generality the color $\{1\}$. Hence, we can color $v$ by the set $\{2,3\}$.

Theorem 2 now readily follows.

Proof of Theorem 2. Let $V(G)=\left\{v_{1}, \ldots, v_{n}\right\}$. For $i \in\{1, \ldots, n\}$, let $f_{v_{i}}: V(G) \rightarrow[3]$ be defined as in Lemma 10 , and let $\varphi_{i}$ be a $\left(3, f_{v_{i}}\right)$-coloring of $G$. Then $\varphi_{1}+\cdots+\varphi_{n}$ is a $(3 n: n+1)$-coloring of $G$. 
Similarly, Lemma 7 implies Theorem 3.

Proof of Theorem 3. Let $D$ be the constant of Lemma 7, let $m=4^{D}$ and let $\delta=\frac{3}{m+1}$. We show that every planar triangle-free graph $G$ of maximum degree at most four and without separating 4-cycles has a $(3 m: m+1)$-coloring, and thus $\chi_{f}(G) \leqslant \frac{3 m}{m+1}=3-\delta$.

Let $G^{\prime}$ be the graph obtained from $G$ by adding edges between all pairs of vertices at distance at most $D-1$. The maximum degree of $G^{\prime}$ is less than $4^{D}=m$, and thus $G^{\prime}$ has a coloring by at most $m$ colors. Let $C_{1}, \ldots, C_{m}$ be the color classes of this coloring (some may be empty). For $i \in[m]$, let $f_{i}$ be the function defined by $f_{i}(v)=2$ for $v \in C_{i}$ and $f_{i}(v)=1$ for $v \in V(G) \backslash C_{i}$. Note that the distance in $G$ between any distinct vertices in $C_{i}$ is at least $D$, and thus Lemma 7 ensures that $G$ has a $\left(3, f_{i}\right)$-coloring $\varphi_{i}$. Then $\varphi_{1}+\cdots+\varphi_{m}$ is a $(3 m: m+1)$-coloring of $G$.

The proof of Theorem 1 is somewhat more involved. Let $G$ be a plane triangle-free graph. We say that $G$ is a counterexample if there exists an integer $n \geqslant|V(G)|$ such that $G$ does not have a $(9 n: 3 n+1)$-coloring. We say that $G$ is a minimal counterexample if $G$ is a counterexample and no plane triangle-free graph with fewer than $|V(G)|$ vertices is a counterexample. Observe that every minimal counterexample is connected.

Lemma 11. If $G$ is a minimal counterexample, then $G$ is 2-connected. Consequently, the minimum degree of $G$ is at least two.

Proof. Let $n \geqslant|V(G)|$ be an integer such that $G$ does not have a $(9 n: 3 n+1)$-coloring. Since $9 n>2(3 n+1)$, it follows that $G$ has at least three vertices. Hence, it suffices to prove that $G$ is 2-connected, and the bound on the minimum degree will follow.

Suppose for a contradiction that $G$ is not 2-connected, and let $G_{1}$ and $G_{2}$ be subgraphs of $G$ such that $G=G_{1} \cup G_{2}$, the graph $G_{1}$ intersects $G_{2}$ in exactly one vertex $v$, and $\left|V\left(G_{1}\right)\right|,\left|V\left(G_{2}\right)\right|<|V(G)|$. By the minimality of $G$, neither $G_{1}$ nor $G_{2}$ is a counterexample, and thus for $i \in\{1,2\}$, there exists a $(9 n: 3 n+1)$-coloring $\varphi_{i}$ of $G_{i}$. By permuting the colors, we can assume that $\varphi_{1}(v)=\varphi_{2}(v)$. Hence, $\varphi_{1} \cup \varphi_{2}$ is a $(9 n: 3 n+1)$-coloring of $G$, which is a contradiction.

Lemma 12. If $G$ is a minimal counterexample, then every face of $G$ has length exactly 5 .

Proof. Let $n \geqslant|V(G)|$ be an integer such that $G$ does not have a $(9 n: 3 n+1)$-coloring. Suppose for a contradiction that $G$ has a face $f$ of length other than 5 . Since $G$ is triangle-free, it has odd girth at least five, and by Lemma 9 , there exists a path $v_{1} v_{2} v_{3}$ in the boundary of $f$ such that the graph $G^{\prime}$ obtained by identifying $v_{1}$ with $v_{3}$ to a single vertex $z$ has odd girth at least five as well. It follows that $G^{\prime}$ is triangle-free. Since $G$ is a minimal counterexample, $G^{\prime}$ has a $(9 n: 3 n+1)$-coloring, and by giving both $v_{1}$ and $v_{3}$ the color of $z$, we obtain a $(9 n: 3 n+1)$-coloring of $G$. This is a contradiction.

Lemma 13. If $G$ is a minimal counterexample, then $G$ has minimum degree at least three.

Proof. Let $n \geqslant|V(G)|$ be an integer such that $G$ does not have a $(9 n: 3 n+1)$-coloring. By Lemma 11, the graph $G$ has minimum degree at least two. Suppose for a contradiction 
that $v \in V(G)$ has degree two. Let $f_{v}$ be defined as in Lemma 10 and let $\varphi_{1}$ be a $\left(3, f_{v}\right)$ coloring of $G$.

Since $G$ is a minimal counterexample and $|V(G-v)| \leqslant n-1$, there exists a tight $(9 n-9: 3 n-2)$-coloring $\varphi_{2}$ of $G-v$. Let $f(x)=3 n-2$ for $x \in V(G-v)$ and $f(v)=3 n-5$. Since both neighbors of $v$ are assigned sets of $3 n-2$ colors, there are at least $(9 n-9)-2(3 n-2)=3 n-5$ colors not appearing at any neighbor of $v$, and thus $\varphi_{2}$ can be extended to a $(9 n: f)$-coloring of $G$.

However, $3 \varphi_{1}+\varphi_{2}$ is a $(9 n: 3 n+1)$-coloring of $G$, which is a contradiction.

Lemma 14. No minimal counterexample contains a safe 5-face.

Proof. Let $G$ be a minimal counterexample. Let $n \geqslant|V(G)|$ be an integer such that $G$ does not have a $(9 n: 3 n+1)$-coloring. Suppose for a contradiction that $f$ contains a safe 5 -face $f=v_{1} v_{2} v_{3} v_{4} v_{5}$, and let $x_{1}, \ldots, x_{4}$ be the neighbors of $v_{1}, \ldots, v_{4}$ that are not incident with $f$, respectively. For $i \in\{1, \ldots, 4\}$, let $f_{v_{i}}$ be defined as in Lemma 10 and let $\varphi_{i}$ be a $\left(3, f_{v_{i}}\right)$-coloring of $G$.

Let $G^{\prime}$ be the plane graph obtained from $G-\left\{v_{1}, v_{2}, v_{3}, v_{4}\right\}$ by identifying $x_{2}$ with $v_{5}$ into a new vertex $u_{1}$, and $x_{3}$ with $x_{4}$ into a new vertex $u_{2}$. Since $f$ is safe, $G^{\prime}$ is trianglefree. Let $N=9 n-54$. Since $G$ is a minimal counterexample and $\left|V\left(G^{\prime}\right)\right| \leqslant n-6$, we conclude that $G^{\prime}$ has a tight $(N: 3 n-17)$-coloring $\varphi_{5}$. Let $f(x)=3 n-17$ for $x \in V\left(G-\left\{v_{1}, v_{2}, v_{3}, v_{4}\right\}\right)$ and $f\left(v_{i}\right)=3 n-20$ for $i \in\{1, \ldots, 4\}$. We extend $\varphi_{5}$ to an $(N, f)$-coloring of $G$ as follows.

Let $\varphi_{5}\left(x_{2}\right)=\varphi_{5}\left(v_{5}\right)=\varphi_{5}\left(u_{1}\right)$ and $\varphi_{5}\left(x_{3}\right)=\varphi_{5}\left(x_{4}\right)=\varphi_{5}\left(u_{2}\right)$. Note that $\mid \varphi_{5}\left(x_{1}\right) \cup$ $\varphi_{5}\left(v_{5}\right) \mid \leqslant 2(3 n-17)$, and thus we can choose $\varphi_{5}\left(v_{1}\right)$ as a subset of $[N] \backslash\left(\varphi_{5}\left(x_{1}\right) \cup \varphi_{5}\left(v_{5}\right)\right)$ of size $3 n-20$. Similarly, choose $\varphi_{5}\left(v_{2}\right)$ as a subset of $[N] \backslash\left(\varphi_{5}\left(x_{2}\right) \cup \varphi_{5}\left(v_{1}\right)\right)$ of size $3 n-20$. Let $M_{3}=[N] \backslash\left(\varphi_{5}\left(v_{2}\right) \cup \varphi_{5}\left(x_{3}\right)\right)$ and $M_{4}=[N] \backslash\left(\varphi_{5}\left(v_{5}\right) \cup \varphi_{5}\left(x_{4}\right)\right)$. Note that $\left|M_{3}\right| \geqslant 3 n-20$ and $\left|M_{4}\right| \geqslant 3 n-20$. Furthermore, since $\varphi_{5}\left(x_{3}\right)=\varphi_{5}\left(x_{4}\right)$ and $\varphi_{5}\left(v_{2}\right) \cap \varphi_{5}\left(v_{5}\right)=\varphi_{5}\left(v_{2}\right) \cap \varphi_{5}\left(x_{2}\right)=\emptyset$, we have $\left|M_{3} \cup M_{4}\right|=N-\left|\varphi_{5}\left(x_{3}\right)\right|=N-(3 n-17)>$ $2(3 n-20)$. Let $\varphi_{5}\left(v_{3}\right) \subseteq M_{3}$ be a set of size $3 n-20$ chosen so that $\left|\varphi_{5}\left(v_{3}\right) \cap M_{4}\right|$ is minimum. Observe that $\left|M_{4} \backslash \varphi_{5}\left(v_{3}\right)\right| \geqslant 3 n-20$, and thus we can choose a set $\varphi_{5}\left(v_{4}\right) \subseteq M_{4} \backslash \varphi_{5}\left(v_{3}\right)$ of size $3 n-20$. This gives an $(N, f)$-coloring of $G$.

Also, by Grötzsch's theorem, $G$ has a $(3: 1)$-coloring $\varphi_{6}$. However, $3\left(\varphi_{1}+\varphi_{2}+\varphi_{3}+\right.$ $\left.\varphi_{4}\right)+\varphi_{5}+6 \varphi_{6}$ is a $(9 n: 3 n+1)$-coloring of $G$, which is a contradiction.

We can now establish Theorem 1.

Proof of Theorem 1. Suppose for a contradiction that there exists a planar triangle-free graph $G$ on $n$ vertices with fractional chromatic number greater than $3-\frac{3}{3 n+1}$. Then $G$ has no $(9 n: 3 n+1)$-coloring, and thus $G$ is a counterexample. Therefore, there exists a minimal counterexample $G_{0}$. Lemmas 13,12 and 8 imply that $G_{0}$ has a safe 5 -face. However, that contradicts Lemma 14. 


\section{References}

[1] M. Albertson, B. Bollobás, and S. Tucker. The independence ratio and the maximum degree of a graph. Congr. Numer., 17:43-50, 1976.

[2] Z. Dvořák, K. Kawarabayashi, and R. Thomas. Three-coloring triangle-free planar graphs in linear time. Trans. on Algorithms, 7:article no. 41, 2011.

[3] Z. Dvořák, D. Král', and R. Thomas. Three-coloring triangle-free graphs on surfaces V. Coloring planar graphs with distant anomalies. arXiv:0911.0885v2, January 2015.

[4] Z. Dvořák and B. Lidický. 3-coloring triangle-free planar graphs with a precolored 8-cycle. J. Graph Theory, forthcoming.

[5] Z. Dvořák and M. Mnich. Large independent sets in triangle-free planar graphs. arXiv:1311.2749, November 2013.

[6] H. Grötzsch. Ein Dreifarbensatz für Dreikreisfreie Netze auf der Kugel. Wiss. Z. Martin-Luther-Univ. Halle-Wittenberg. Math.-Nat. Reihe, 8:109-120, 1958/1959.

[7] A. Hilton, R. Rado, and S. Scott. A $(<5)$-colour theorem for planar graphs. Bull. London Math. Soc., 5:302-306, 1973.

[8] K. F. Jones. Minimum independence graphs with maximum degree four. In Graphs and applications (Boulder, Colo., 1982), Wiley-Intersci. Publ., pages 221-230. Wiley, 1985.

[9] W. Klostermeyer and C. Q. Zhang. $(2+\epsilon)$-coloring of planar graphs with large odd-girth. J. Graph Theory, 33:109-119, 2000.

[10] Ł. Kowalik. Fast 3-coloring triangle-free planar graphs. In Susanne Albers and Tomasz Radzik, editors, ESA, volume 3221 of Lecture Notes in Computer Science, pages 436-447. Springer, 2004.

[11] E. R. Scheinerman and D. H. Ullman. Fractional Graph Theory. Dover Publications Inc., Mineola, NY, 2011.

[12] R. Steinberg and C. A. Tovey. Planar Ramsey numbers. J. Combin. Theory, Ser. B, 59(2):288-296, 1993.

[13] C. Thomassen. Grötzsch's 3-color theorem and its counterparts for the torus and the projective plane. J. Combin. Theory, Ser. B, 62:268-279, 1994.

[14] C. Thomassen. A short list color proof of Grötzsch's theorem. J. Combin. Theory, Ser. B, 88:189-192, 2003. 